\section{Perfil físico funcional de indivíduos atendidos em um centro de atenção psicossocial: importância da inserção fisioterapêutica}

\section{Functional physical profile of individuals attended in a psychosocial care center: importance of physiotherapeutic insertion}

Luana Carvalho, Polliana Radtke dos Santos, Angela Cristina Ferreira da Silva, Éboni Marília Reuter

RESUMO

Introdução: Em geral, indivíduos com transtornos mentais apresentam dificuldade no autocuidado, o que pode levar ao aparecimento ou agravamento de outras morbidades. Objetivo: Identificar evidências da importância da atuação fisioterapêutica na atenção de indivíduos com transtornos mentais, através de alterações físico-funcionais em participantes de oficinas ofertadas por um Centro de Atenção Psicossocial. Métodos: Estudo quantitativo transversal avaliou 15 sujeitos. Foram realizados os seguintes testes: Teste de Sentar e Alcançar, dinamometria de preensão palmar, Escala Visual Analógica, Teste de Romberg, Timed Up and Go, index-index, index-nariz, index-nariz-dedo, joelhocalcanhar e goniometria. As análises das variáveis de alterações físico funcionais foram realizadas em mediana e intervalo interquartil para dados contínuos e frequência absoluta para dados categóricos. Resultados: Na avaliação da flexibilidade, as categorias encontradas foram 'precisa melhorar' $(n=$ doze) e 'regular' ( $n=$ três). Oito participantes relataram dores corporais. No equilíbrio estático, dois terços dos sujeitos apresentaram oscilações durante a manobra, enquanto no equilíbrio dinâmico um participante apresentou resultado que indica necessidade de intervenção. Para coordenação motora, pelo menos dois terços dos sujeitos apresentaram positivação no teste e na goniometria metade dos sujeitos apresentaram alguma redução de amplitude de movimentos. Conclusão: Considerando as alterações físico funcionais, especialmente de flexibilidade, equilíbrio e coordenação motora, fica evidente a importância da inclusão do fisioterapeuta em serviços que atendem sujeitos com transtornos mentais.

PALAVRAS-CHAVE: Transtornos mentais; Serviços de Saúde Mental; Fisioterapia.

\section{ABSTRACT}

Introduction: In general, individuals with mental disorders have difficulty in self-care, which can lead to the appearance or aggravation of other morbidities. Objective: To identify evidence of the importance of physical therapy in the care of individuals with mental disorders through physical-functional changes in workshop participants offered by a Psychosocial Care Center. Methods: Cross - sectional quantitative study evaluated 15 subjects. The following tests were performed: Sit and Reach Test, palmar grip dynamometry, Visual Analog Scale, Romberg Test, Timed Up and Go, index-index, indexnose, finger-nose index, knee-heel and goniometry. Analyzes of functional physical change variables were performed at median and interquartile range for continuous data and absolute frequency for categorical data. Results: In the assessment of flexibility, the categories found were 'need to improve' ( $n=$ twelve) and 'regular' ( $n=$ three). Eight participants reported body aches. In the static equilibrium, two thirds of the subjects presented oscillations during the maneuver, while in the dynamic equilibrium one participant presented an outcome indicating the need for intervention. For motor coordination, at least two-thirds of the subjects had a positive test and in the goniometry half of the subjects had some reduction in their range of motion. Conclusion: Considering the functional physical changes, especially of flexibility, balance and motor coordination, it is evident the importance of the inclusion of physiotherapist in services that attend subjects with mental disorders.

KEYWORDS: Mental Disorders; Mental Health Services; Physical Therapy 


\section{INTRODUÇÃO}

Os transtornos mentais podem se apresentar de formas diferentes nas relações de classes e gêneros, envolver dimensões econômicas, sociais, políticas e culturais'. Em geral indivíduos com transtornos mentais apresentam dificuldade no autocuidado, o que pode levar ao aparecimento ou agravamento de outras morbidades. As tensões diárias tendem a gerar sintomas que podem estar relacionados com a condição mental².

Entre estes sintomas destacam-se as limitações físicas sendo uma delas na estrutura corporal e no movimento, desenvolvendo modificações posturais, tensões musculares, rigidez e dificuldade na execução dos movimentos alterações do padrão respiratório 3,4

Considerando estas possíveis inadequações, o fisioterapeuta apresenta uma atuação importante na prevenção e promoção da saúde, englobando os aspectos físicos e mentais. 0 sedentarismo, medicamentos e outros fatores influenciam nas inúmeras queixas físicas dos indivíduos portadores de transtornos mentais. 0 que demonstra a importância do acesso ao atendimento fisioterapêutico por essa população $0^{5,6}$. A partir do exposto, o objetivo foi identificar o perfil físico-funcional de participantes das oficinas ofertadas por um Centro de Atenção Psicossocial, evidenciando a necessidade do fisioterapeuta neste serviço.

\section{MÉTODO}

\section{Delineamento, cenário, amostra e aspectos éticos}

Trata-se de estudo quantitativo transversal, realizado em um Centro de Atenção Psicossocial (CAPS) do Sul do Brasil'. O período de coleta de dados foi no mês de fevereiro de 2017. A amostra não-probabilística e intencional incluiu indivíduos atendidos na modalidade intensiva, portadores de transtornos mentais graves e persistentes, que fossem participantes das oficinas terapêuticas oferecidas pelo serviço.

Os participantes foram escolhidos de acordo com os seguintes critérios de inclusão: indivíduos adultos, com idade entre 18 e 60 anos, que utilizassem dos serviços prestados pelo referido CAPS, que fossem participantes das oficinas oferecidas e estivessem de acordo em participar da pesquisa. Foram excluídos do estudo indivíduos apresentando algum trauma musculoesquelético recente que pudessem alterar as variáveis a serem avaliadas, ou que não possuíssem capacidade cognitiva para entender a execução dos testes.

O projeto foi apreciado pelo Comitê de Ética e Pesquisa (CEP) da Universidade de Santa Cruz do Sul - UNISC parecer número 1640694, sendo o aceite dos indivíduos que concordaram em participar obtido através do Termo de Consentimento Livre e Esclarecido.

\section{Avaliação físico-funcional}

Para caracterização da amostra foi realizada anamnese, obtendo-se dados demográficos, como sexo, idade, profissão, tempo de acompanhamento no CAPS, diagnóstico clínico, medicamentos e doenças associadas. Em seguida iniciou-se a aplicação de testes específicos para avaliação físico-funcional.

Para avaliação da saúde musculoesquelética, foi aplicado o Teste de Sentar e Alcançar, o qual foi executado com o Banco de Wells, seguindo roteiro de Bertolla et al $\left.\right|^{8}$. Foram realizadas três medidas expressadas em centímetros $(\mathrm{cm})$, sendo que a medida de maior valor foi a validada e classificada conforme critérios de Heyward9. Para força muscular, a dinamometria de preensão palmar foi efetuada conforme protocolo de Dias et al ${ }^{10}$, com dinamômetro Jamar. Valores obtidos apenas do membro superior dominante, registrados em quilogramas força (Kgf).

Os sujeitos também foram questionados quanto a dor através da Escala Visual Analógica. Na presença de dor, foi pedido para que o participante indicasse quantitativamente a que estava sentindo no momento, bem como a localização da mesma.

Para avaliação do equilíbrio, aplicou-se o Teste de Romberg na avaliação estática, seguindo protocolo de Bechara e Santos ${ }^{11}$. 0 teste foi sensibilizado por algumas manobras, entre elas a de pedir para que o participante colocasse um pé a frente do outro. Considerado positivo quando ocorreu piora do equilíbrio e tendência à queda dentro do tempo de um minuto. Para equilíbrio dinâmico, aplicou-se o Timed Up and Go (TUG), realizado e classificado conforme roteiro de Almeida et $a^{12}$.

Ao avaliar a coordenação motora foram executados os testes de índex-índex, índex-nariz, dedo-nariz-dedo e joelho-calcanhar, conforme protocolo descrito por O'Sullivan e Schimitz ${ }^{13}$, sendo considerado positivo quando houve algum desvio da posição durante o teste, ou quando ocorreu diminuição na qualidade da resposta com os olhos fechados.

Por fim, foram avaliadas as amplitudes de movimento por goniometria das articulações de ombro, cotovelo, punho, quadril, joelho e tornozelo, com a utilização de um goniômetro universal, seguindo protocolo de Norkin e White ${ }^{14}$ e também suas classificações para os limiares de amplitude de movimento de cada articulação.

\section{Métodos estatísticos}

As análises das variáveis de alterações físico funcionais foram realizadas em mediana e intervalo interquartil para dados contínuos e frequência absoluta para dados categóricos.

\section{RESULTADO}

Aamostra composta por quinze participantes possui as seguintes características sociodemográficas: oito homens, mediana de idade de 44 anos (intervalo interquartil 34-53 anos). Dez possuíam algum tipo de benefício da previdência 

sujeito atuando como babá.

Quanto às características clínicas, pode-se considerar que $75 \%$ dos sujeitos eram vinculados ao CAPS há mais de cinco anos. 0 diagnóstico clínico, identificou-se os seguintes conjuntos de transtornos: afetivo bipolar ( $\mathrm{n}=$ quatro), depressivo ( $n=$ três), distúrbio de atividade e atenção $(n=u m)$, esquizoafetivo $(n=u m)$, esquizofrenia $(n=$ dois), mental não especificado ( $n$ = três) e orgânico não especificado ( $n$ = dois). Todos faziam uso de medicação, com mediana de três medicamentos por pessoa. Dos medicamentos em uso, destaca-se o ácido valpróico, biperideno, clorpromazina olanzapina e risperidona. Outros medicamentos se referem a tratamento para doenças associadas, como atenolol $(n=$ dois), levotiroxina, omeprazol, sinvastatina e sulfato ferroso ( $n=$ um cada) (Tabela 1).

Tabela 1. Características clínicas de sujeitos com transtornos mentais participantes das oficinas do Centro de Atenção Psicossocial (CAPS)

\begin{tabular}{ll}
\hline Variáveis & $\mathrm{n}$ - $\mathrm{md}(\mathrm{IQ})$ \\
\hline Tempo de vínculo CAPS (anos) md (IQ) & $12(5-16)$ \\
Medicamentos ( $\mathrm{n})$ & 15 \\
Sim & - \\
Não & $3(3-5)$ \\
Quantidade de medicamento por pessoa md (IQ) & \\
Tipo de medicamento (n) & 8 \\
Ácido valpróico & 8 \\
Biperideno & 2 \\
Clonazepan & 4 \\
Clorpromazina & 1 \\
Clozapina & 2 \\
Diazepam & 1 \\
Fenobarbital & 1 \\
Flufenazina & 1 \\
Fluoxetina & 3 \\
Haloperidol & 1 \\
Lítio & 3 \\
Olanzapina & 1 \\
\hline
\end{tabular}

Quetiapina

Topiramato

Doenças associadas (n)

Sim

Não

Tipo de doenças (n)

Anemia

Diabetes Mellitus

Epilepsia não especificada

Hipertensão Arterial Sistêmica

Hipertireoidismo

md: mediana; IQ: intervalo interquarti

Fonte: arquivo da pesquisadora, 2017

Na avaliação da flexibilidade, as categorias encontradas foram 'precisa melhorar' $(n=$ doze e e 'regular' $(n=$ três). Não houveram sujeitos classificados nas categorias 'bom', 'muito bom' e 'excelente'. Na avaliação da dor, oito participantes relataram dor, sendo a localização mais citada a região lombar $(n=$ quatro). Na avaliação da força muscular, metade dos sujeitos atingiram nove Kgf. Nos testes de coordenação motora, pelo menos dois terços dos sujeitos apresentaram positivação no teste, o que indica déficit (Tabela 2).

$\mathrm{Na}$ avaliação do equilibrio, maiores alterações foram encontradas quando o teste foi estático, sendo que dois terços dos sujeitos apresentaram oscilações durante a manobra. Já quando avaliados na forma dinâmica, apenas um participante apresentou resultado que indica necessidade de intervenção (Tabela 2).

Tabela 2. Avaliação físico-funcional de sujeitos com transtornos mentais. Flexibilidade, dor, força de preensão palmar, coordenação motora, equilíbrio estático e dinâmico

\begin{tabular}{ll}
\hline Variáveis & $\mathrm{n}$ - md (IQ) \\
\hline Teste de Sentar e Alcançar (cm) md (IQ) & $12(7-24)$ \\
Classificação Teste de Sentar e Alcançar ( $\mathrm{n})$ & \\
$\quad$ Precisa melhorar & 12 \\
Regular & 3 \\
\hline ISSN 2236-5834 & 5
\end{tabular}


Escala Visual Analógica (n)

Sim

Escala Visual Analógica valor md (IQ)

lização (n)

Região lombar

Punho direito

Membros inferiores

Joelho esquerdo

Dinamometria (kgf) $\mathrm{md}(\mathrm{IQ})$

\section{Index-index (n)}

Positivo

Negativo

Index-nariz (n)

Positivo

Negativo

Index-nariz-dedo (n)

Positivo

Negativo

Joelho-calcanhar ( $\mathrm{n})$

Positivo

Negativo

Romberg (n)

Positivo

Negativo

Timed Up and Go (seg) md (IQ)

Classificação Timed Up and Go (n)

Sem alterações

Não há evidencias significativas

Necessidade de intervenção

md: mediana; IQ: intervalo interquartil,

Na goniometria, percebe-se que metade dos sujeitos apresentaram alguma redução de determinados movimentos, sendo essa na amplitude de rotação medial com 33\% e 50\% na extensão de ombro, 37\% na flexão e 42\% na extensão de punho, $33 \%$ na flexão, $50 \%$ na extensão, $55 \%$ na rotação medial e lateral de quadril, $40 \%$ na flexão plantar e $42 \%$ na inversão de tornozelo. Quando comparados os valores entre hemicorpos, as medianas e intervalos interquartis foram os mesmos para flexão e extensão de ombro, extensão de cotovelo, adução de punho, extensão abdução, adução e rotação externa de quadril, flexão plantar, inversão e eversão de tornozelo (Tabela 3).

Tabela 3. Avaliação fisico-funcional de sujeitos com transtornos mentais. Amplitude de movimento

$9(4-20)$

10

\begin{tabular}{|c|c|c|}
\hline Articulações & $\begin{array}{l}\text { Direito } \\
\mathrm{md}(\mathrm{IQ})\end{array}$ & $\begin{array}{l}\text { Esquerdo } \\
\mathrm{md}(\mathrm{IQ})\end{array}$ \\
\hline \multicolumn{3}{|l|}{ Ombro } \\
\hline Flexão & $160(150-170)$ & $160(150-170)$ \\
\hline Extensão & $30(20-30)$ & $30(20-30)$ \\
\hline Abdução & $130(120-160)$ & $130(120-150)$ \\
\hline Adução & $130(120-160)$ & $130(120-150)$ \\
\hline Rotação medial & $60(40-70)$ & $60(50-70)$ \\
\hline Rotação lateral & $60(50-90)$ & $60(50-70)$ \\
\hline \multicolumn{3}{|l|}{ Cotovelo } \\
\hline Flexão & $120(110-130)$ & $130(110-130)$ \\
\hline Extensão & $120(110-130)$ & $120(110-130)$ \\
\hline \multicolumn{3}{|l|}{ Punho } \\
\hline Flexão & $50(30-60)$ & $40(30-50)$ \\
\hline Extensão & $40(30-50)$ & $30(30-50)$ \\
\hline Desvio ulnar & $20(20-30)$ & $20(20-20)$ \\
\hline Desvio radial & $20(20-20)$ & $20(20-20)$ \\
\hline \multicolumn{3}{|l|}{ Quadril } \\
\hline Flexão & $80(60-90)$ & $80(60-80)$ \\
\hline Extensão & $15(10-30)$ & $15(10-30)$ \\
\hline Abdução & $30(20-30)$ & $30(20-30)$ \\
\hline Adução & $30(20-30)$ & $30(20-30)$ \\
\hline Rotação medial & $20(20-23)$ & $20(20-30)$ \\
\hline
\end{tabular}


Rotação lateral

$20(20-30)$

$20(20-30)$

Joelho

Flexão

$120(100-130)$

$110(100-123)$

Extensão

$120(100-130)$

$110(100-123)$

Tornozelo

$\begin{array}{lll}\text { Flexão plantar } & 30(20-30) & 30(20-30) \\ \text { Flexão dorsal } & 20(10-30) & 20(10-20) \\ \text { Inversão } & 20(20-20) & 20(20-20) \\ \text { Eversão } & 20(10-20) & 20(10-20)\end{array}$

md: mediana; IQ: intervalo interquartil;

Fonte: arquivo da pesquisadora, 2017.

\section{DISCUSSÃO}

Percebeu-se que o perfil físico-funcional da presente amostra de pessoas com transtornos mentais evidenciou alterações especialmente na flexibilidade, equilibrio estático e coordenação motora. Ainda, redução da amplitude de movimento de determinadas articulações e presença de dores corporais. Estes achados evidenciam a necessidade do fisioterapeuta em unidades de saúde mental, buscando projetos terapêuticos atentos a estas informações.

Ressalta-se que os resultados são importantes na medida em que permitem ao serviço planejar ações, especialmente por se tratar de pacientes em acompanhamento intensivo. Embora tenha-se avaliado todos os sujeitos aptos a executarem os testes, considera-se que o número reduzido da amostra pode ser um fator limitante. A variedade de diagnósticos e medicamentos também não nos permitiram fazer uma relação comparativa com as alterações funcionais. Entretanto, sabe-se que é característica de um serviço que atende um amplo espectro de transtornos, pois assim como os diagnósticos são variados, os medicamentos também serão, de acordo com os sintomas de cada indivíduo.

Os sujeitos desta pesquisa faziam uso de diversos medicamentos, entre eles os mais citados são os antipsicóticos. Em uma pesquisa realizada por Barbosa e Silva ${ }^{15}$, destaca-se que essa classe medicamentosa apresenta efeitos positivos ao tratamento dos transtornos mentais, pois reduzem delírios e alucinações. Em contrapartida, podem provocar efeitos no sistema musculoesquelético, sendo os mais comuns, disfunções crônicas como ataxia, atrofia, fraqueza muscular, entre outros que podem afetar diretamente a capacidade funcional.

Ainda, os autores indicam que indivíduos com esquizofrenia apresentam alteração no equilibrio dinâmico, o que pode influenciar na diminuição ou dificuldades funcionais, sendo o aumento do risco de quedas relacionado com os efeitos colaterais causados pelos antipsicóticos ${ }^{15}$. Estes efeitos são sentidos pelos usuários, uma vez que entre as queixas mais frequentes em indivíduos com diagnóstico de depressão, esquizofrenia, transtorno obsessivo compulsivo e de ansiedade são as dores musculares, alterações posturais e de equilíbrio, dispneia na realização de esforços e dificuldade na deambulação ${ }^{16}$. No nosso estudo os indivíduos também apresentaram dores corporais e alteração no equilíbrio, especialmente quando avaliados na forma estática, o que também pode relacionar-se com demais alterações funcionais.

Indivíduos com esquizofrenia e transtorno bipolar apresentam sinais neurológicos sutis relacionados as funções de integração social, coordenação motora, sequenciamento de tarefas motoras complexa e também reflexo primitivos. Em um estudo que realizou comparação entre um grupo controle e indivíduos com transtorno de humor, observou-se que no último há maiores disfunções motoras e sensoriais, sendo que tais alterações também são identificadas em esquizofrênicos ${ }^{17,18}$. No nosso estudo os indivíduos avaliados também apresentaram déficits na coordenação motora, indo ao encontro da literatura, e evidenciando a necessidade de intervenção.

Na literatura atual, não identificamos autores que falem diretamente sobre a amplitude de movimento em indivíduos com transtornos mentais. No entanto os indivíduos avaliados neste estudo apresentaram alterações significativas na flexibilidade, o que pode relacionar-se diretamente na diminuição da amplitude de movimento de determinadas articulações. Em estudo realizado com idosos, os autores ressaltam que, além da redução da produção do movimento realizado por uma articulação, a alteração na flexibilidade pode aumentar a probabilidade de lesões nas articulações e músculos envolvidos no movimento. Também pode ocorrer aumento no risco de quedas, impacto no desempenho funcional, alterações de equilíbrio, postura, problemas respiratórios, dificuldade nas atividades de vida diária, alteração no padrão da marcha, relacionado com a perda de força associada a diminuição da flexibilidade ${ }^{19,20}$

Em um projeto de atuação fisioterapêutica realizado em um CAPS Regional III do Ceará, realizou-se intervenções baseadas na promoção do movimento corporal, flexibilidade, estabilidade, cognição e interação entre os indivíduos, tendo como resultados o relato de sensação de bem-estar e maior disposição para a realização das atividades de vida diária²1. Nesse sentido, ações de reabilitação por meio de exercício terapêutico para indivíduos com transtornos mentais indicam que os participantes inicialmente apresentam certa dificuldade de interagir socialmente, mas conforme identificam suas fragilidades, passam a apresentar melhora geral. Estes aspectos materializam a importância de realizar práticas de prevenção e terapias voltadas para esta população de forma a minimizar as possíveis alterações musculoesqueléticas ${ }^{22}$

A fisioterapia apresenta um papel importante neste contexto de prevenção e complicações dos agravos, promoção da saúde e recuperação da função ${ }^{15}$. Mesmo que na literatura a atuação do fisioterapeuta na saúde mental ainda é escassa, os resultados disponíveis mostram efeitos importantes, trazendo benefícios fisiológicos, psicológicos e funcionais ${ }^{21}$

ISSN 2236-5834 


\section{CONSIDERAÇÕES FINAIS}

Ficou evidenciado alterações físico-funcionais em participantes intensivos de um CAPS do sul do Brasil. Tais alterações incluíram redução de flexibilidade, amplitude de movimento, alterações de equilíbrio estático, coordenação motora e presença de dor corporal. A inserção fisioterapêutica em serviços de atenção à saúde mental mostra-se necessária, atuando na prevenção e recuperação de possíveis morbidades advindas do próprio transtorno mental ou dos medicamentos utilizados por esta população.

O fisioterapeuta como profissional da saúde que usa o movimento para avaliar e desenvolver a funcionalidade tem como premissa a qualidade de vida dos sujeitos por ele assistidos. Portanto, os participantes do CAPS lograriam benefícios no âmbito da função, da diminuição de algias e aumento significativo da flexibilidade, o que acarretaria melhora da autoestima e do desenvolvimento para o autocuidado.

Dentro deste contexto evidencia-se também a necessidade divulgar os achados à gestão do município e equipe do CAPS em que a pesquisa foi executada. Tais dados, os quais são proveniente de medidas objetivas, subsidiam a prática dos profissionais e permitem traçar estratégias que objetivem reduzir e prevenir as referidas alterações fisícofuncionais

\section{REFERÊNCIAS}

1. Quadros LCM, Laura HC, Quevedo LA, Gigante DP. Efeitos da mobilidade social na saúde mental de adultos: uma revisão sistemática da literatura. Cien Saude Colet. 2016;21(2):443. DOI: 10.1590/1413-81232015212.17942014

2. Branquinho JS, Gomes FA, Silva RP, Leite MMA, Candido ALS, Lima LA, et al. Doenças crônicas em pacientes com transtornos mentais. Gestão e Saude. 2014;5:2458-2464.

3. Ruo B, Baker DW, Thonpson JA, Murray PK, Huber GM, Sudano Jr JJ. Patients with worse mental health report more physical limitations after adjustment for physical performance. Psychosom Med. 2008;70(4):417-421. DOI: 10.1097/ PSY.0b013e31816f858d

4. Pauli K, Campos R. A inserção do fisioterapeuta na equipe multiprofissional do centro de atenção psicossocial. Fisiot Saude Func. 2016;5(1):14-22.
5. Australian Physiotherapy Association. Position statement of mental health and physiotherapy [Internet]. 2016. Available from httpwww.physiotherapy.asn.au

6. Probst M. Physiotherapy and Mental Health. In: Clinical Physical Therapy. InTech, 2017. DOI: http://dx.doi. org/10.5772/67595

7. Goldim JN. Manual de iniciação à pesquisa em saúde. 2a ed. rev e ampl Porto Alegre: Decasa; 2000.

8. Bertolla F, Baroni BM, Leal Junior ECP, Oltramari JD. Efeito de um programa de treinamento utilizando o método Pilates $®$ na flexibilidade de atletas juvenis de futsal. Rev Bras Med Esporte. 2007;13(4):222-6.

9. Heyward VH. Avaliação física e prescrição de exercício. 6. ed. Porto Alegre: Artmed; 2013.

10. Dias JA, Ovando AC, Külkamp W, Junior NGB. Força de preensão palmar: métodos de avaliação e fatores que influenciam a medida. Rev Bras Cineantropom. Desempenho Hum. 2010;12(3):209-16.

11. Bechara FT, Santos SMS. Efetividade de um programa fisioterapêutico para treino de equilibrio em idosos. Rev Saude Pesq. 2008;1(1):15-20

12. Almeida ST, Soldera CLC, Carli GA, Gomes I, Resende TL. Análise de fatores extrínsecos e intrínsecos que predispõem a quedas em idosos. Rev Assoc Med Bras. São Paulo. 2012;58(4):427-433.

13. O’sullivan SB, Schimitz TJ. Fisioterapia: avaliação e tratamento. 2a ed. São Paulo: Manole; 1993.

14. Norkin CC, White J. Medida do movimento articular: manual de goniometria. 2a ed. Porto Alegre: Artes Médicas; 1997

15. Barbosa ÉG, Silva EAM. Fisioterapia na saúde mental: uma revisão de literatura. Ver Saud Física e Mental. 2014;3(2):12-30

ISSN 2236-5834 
16. Silva RCB, Balk TMCS, Santos FM, Jesien S. Grupo de movimento terapêutico, o exercício físico como estratégia de intervenção na saúde mental. Anais do Salão Internacional de Ensino, Pesquisa e Extensão. 2015;7(4).

17. Pedroso VSP, Salgado JV, TeixeiraAL. Sinais neurológicos sutis: uma revisão. J. bras. psiquiatr. 2010;59(3):233237. DOI: DOI: 10.1590/S0047-20852010000300010

18. Chan RC, Gottesman II. Neurological soft signs as candidate endophenotypes for schizophrenia: a shooting star or a Northern star? Neurosci Biobehav Rev. 2008;32(5):957-71. DOI: 10.1016/j.neubiorev.2008.01.005

19. Spirduso WW. Physical dimensions of aging. Champaign, IL: Human Kinetics; 1995.

20. Fidelis LT, Patrizzi LJ, Walsh IAP. Influência da prática de exercícios físicos sobre a flexibilidade, força muscular manual e mobilidade funcional em idosos. Rev Bras Geriatr gerontol. 2013;16(1):109-16.

21. Jesus M, Resende F, Nunes ACL. Cuidado em saúde mental: perspectiva de atuação da fisioterapêutica. Fisiot Saude Func. 2013;2(1):3-5.

22. Lima ITLG, Mundim FSP. Enfoque fisioterapêutico na promoção de saúde para pacientes portadores de transtornos mentais: projeto saber viver -um relato de experiência. Cienc Contemporânea. 2016;1(1):61-71. 